\title{
640-Gbit/s data transmission and clock recovery using an ultrafast periodically poled lithium niobate device
}

Oxenløwe, Leif Katsuo; Gomez-Agis, F.; Ware, C.; Kurimura, S.; Mulvad, Hans Christian Hansen; Galili, Michael; Nakajima, H.; Ichikawa, J.; Erasme, D.; Clausen, Anders

Total number of authors:

11

Published in:

Journal of Lightwave Technology

Link to article, DOI:

10.1109/JLT.2008.2009322

Publication date:

2009

Document Version

Publisher's PDF, also known as Version of record

Link back to DTU Orbit

Citation (APA):

Oxenløwe, L. K., Gomez-Agis, F., Ware, C., Kurimura, S., Mulvad, H. C. H., Galili, M., Nakajima, H., Ichikawa, J., Erasme, D., Clausen, A., \& Jeppesen, P. (2009). 640-Gbit/s data transmission and clock recovery using an ultrafast periodically poled lithium niobate device. Journal of Lightwave Technology, 27(3), 205-213.

https://doi.org/10.1109/JLT.2008.2009322

\section{General rights}

Copyright and moral rights for the publications made accessible in the public portal are retained by the authors and/or other copyright owners and it is a condition of accessing publications that users recognise and abide by the legal requirements associated with these rights.

- Users may download and print one copy of any publication from the public portal for the purpose of private study or research.

- You may not further distribute the material or use it for any profit-making activity or commercial gain

- You may freely distribute the URL identifying the publication in the public portal 


\title{
640-Gbit/s Data Transmission and Clock Recovery Using an Ultrafast Periodically Poled Lithium Niobate Device
}

\author{
Leif Katsuo Oxenløwe, Fausto Gómez-Agis, Cédric Ware, Sunao Kurimura, Hans Christian H. Mulvad, \\ Michael Galili, Hirochika Nakajima, Junichiro Ichikawa, Didier Erasme, Anders Thomas Clausen, and \\ Palle Jeppesen, Member, IEEE
}

\begin{abstract}
This paper presents the first demonstration of the use of a periodically poled lithium niobate device for signal processing at $640 \mathrm{Gbit} / \mathrm{s}$. Clock recovery is performed successfully using the lithium niobate device, and the clock signal is used to control a nonlinear fiber-based demultiplexer. The full 640-Gbit/s system gives error-free performance with no pattern dependence and there is less than 1-dB power penalty after 50-km fiber transmission.
\end{abstract}

Index Terms-Clock recovery, optical fiber communication, optical switches, ultrahigh-speed data transmission.

\section{INTRODUCTION}

$\mathbf{T}$ HE demand for higher telecommunication bandwidth is continuously growing, and the challenge of providing it is met in research labs around the world by strong efforts to find new techniques and technologies to lift the burden. For instance, an impressive 25.6-Tbit/s data transmission using polarization multiplexed RZ-DQPSK in the $\mathrm{C}+\mathrm{L}$ band was demonstrated recently [1]. In addition, at OFC 2008, plenary speaker Bob Metcalfe, inventor of the Ethernet, professed that 1 Terabit/s Ethernet (TE) will be needed in the near future and that it is essential to conduct fundamental research on new technologies to enable this, since current technologies cannot [2]. Whether $1 \mathrm{TE}$ will be best created by a serial or a parallel approach is an open question, but to answer it, it is necessary to conduct research on multiple paths.

Manuscript received June 29, 2008; revised October 10, 2008. Current version published February 13, 2009. This work was supported by the Danish Research Council-funded project NANO-COM, the European COST-291 and 288 programs, the e-Photon One Network of Excellence, CONACyT-Mexico, OFS Fitel Denmark, and NiCT, Japan.

L. K. Oxenløwe, H. C. H. Mulvad, M. Galili, A. T. Clausen, and P. Jeppesen are with DTU Fotonik, Technical University of Denmark, DK-2800 Lyngby, Denmark (e-mail: leif.oxenlowe@ fotonik.dtu.dk; lkox@ fotonik.dtu.dk; hchm@com.dtu.dk; mg@com.dtu.dk; atc@com.dtu.dk; pj@com.dtu.dk).

F. Gómez-Agis, C. Ware, and D. Erasme are with the Communications and Electronics Department, Institut TELECOM, TELECOM ParisTech, LTCI CNRS, 75634 Paris Cedex 13, France (e-mail: fausto.gomez@telecom-paristech.fr; cedric.ware@ telecom-paristech.fr; didier.erasme @ telecom-paristech.fr).

S. Kurimura is with National Institute for Materials Science, Tsukuba 3050044, Japan (e-mail: kurimura.sunao@nims.go.jp).

H. Nakajima is with Graduate School of Science and Technology, Waseda University, Tokyo 169-8555, Japan (e-mail: hiro@ pic.phys.waseda.ac.jp).

J. Ichikawa is with New Technology Research Labs, Sumitomo Osaka Cement Company Ltd., Chiba 274-8601, Japan (e-mail: ichikawa@opto.soc.co. jp).

Color versions of one or more of the figures in this paper are available online at http://ieeexplore.ieee.org.

Digital Object Identifier 10.1109/JLT.2008.2009322
One path to increase the channel capacity is to increase the serial data rate. To go far beyond $100 \mathrm{Gbit} / \mathrm{s}$, which is the current electrical state-of-the-art, e.g., [3], will require optical techniques. Using optical time-division multiplexing (OTDM), 640 Gbaud symbol rates has so far been demonstrated as the highest pulse rate carrying data by a few groups worldwide, first in [4] and then most notably in [5]. For almost twenty years now, OTDM has been explored as a possible route to generate high bit rates in the optical domain, but there has hitherto been no market penetration. There are several reasons for this, and apart from market circumstances, we believe the most important one is the lack of good, stable and practical solutions for essential functionalities. With the introduction of internet video transmission, the bit rates have exploded, and internet exchange office congestion is becoming a real limitation. Therefore, there is once again a need for basic research in solutions to congestion problems. There is currently a great focus on $100-\mathrm{Gb} / \mathrm{s}$ Ethernet (100 GE), and, five to ten years from now, in Internet exchange stations, one may have several $100 \mathrm{GE}$ lines, which may need to be transmitted to the same destination and to avoid congestion, it may be beneficial to employ an ultrafast optical Ethernet multiplexing. This would result in an optical $1000 \mathrm{GE}$, or 1 Terabit/s Ethernet, 1 TE. One great concern with the parallel technologies developed so far is their massive power consumption. Serial solutions combined with circuit switched networks may help to reduce the power consumption. An essential functionality, very challenging at these ultrahigh bit rates, is that of clock recovery (CR), and it was not until very recently (2007) that this was demonstrated for the first time at 640 Gbaud [6], breaking the 1996 record of $400 \mathrm{Gbit} / \mathrm{s}$ [7]. Filter-assisted cross-phase modulation in a semiconductor optical amplifier (SOA) [8] was used, and this allowed for the 640-Gbit/s clock recovery [6], [9]. However, in addition to the fast effects in semiconductors, they also have slow recovery times, which will inevitably lead to patterning effects for OOK modulation formats. To avoid this, a truly ultrafast scheme would be preferable. Nonlinear wave mixing in lithium niobate is one such scheme, as proposed in [10]. In fact over the last few years, periodically poled lithium niobate (PPLN) has proven to be a very promising candidate for a key component in optical signal processing [11], [12].

This paper reports on the use of a truly ultrafast, and thus pattern-independent, all-optical nonlinear effect for CR, namely sum-frequency generation in a quasi-phase-matched (QPM) adhered ridge waveguide periodically poled $\mathrm{LiNbO} 3$ module (ARW PPLN), and demonstrates its potential in a successful 


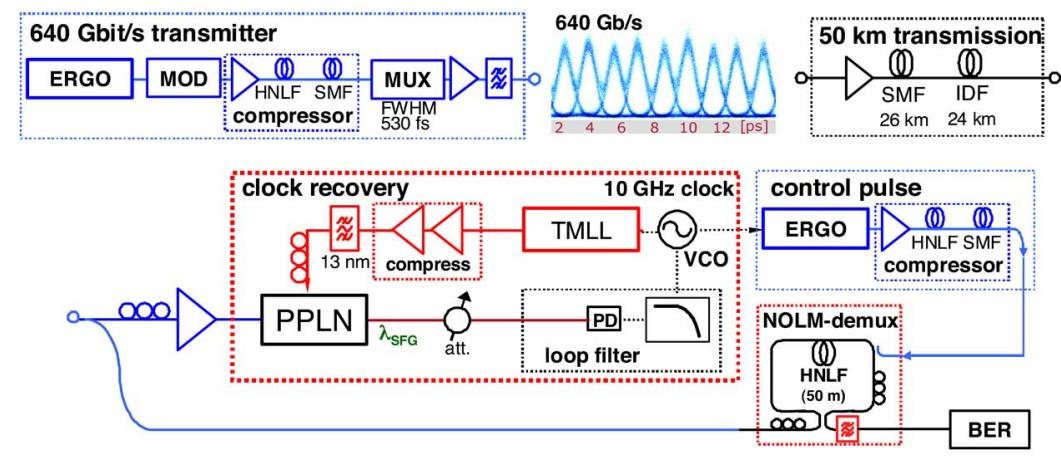

Fig. 1. Setup for 640-Gbit/s transmission, clock recovery, and demultiplexing.

640-Gbit/s transmission experiment. This is the first time PPLN is demonstrated at such high bit rates and the second time ever that a full 640-Gbit/s transmission experiment, i.e., including clock recovery on the aggregated line rate data signal, is conducted. In this case, no pattern dependence is theoretically expected, nor experimentally found. Error free and low-penalty 640-Gbit/s transmission is obtained and the PPLN requires only 1-mW average 640-Gbit/s data power.

\section{EXPERIMENTAL PROCEDURE}

The experimental setup used is sketched in Fig. 1. The optical signal is generated by an erbium glass mode-locked laser (ERGO) at $10 \mathrm{GHz}$ and $1557 \mathrm{~nm}$. The pulses are data modulated with a $2^{7}-1$ PRBS (MOD), and after pulse compression multiplexed in a passive fiber-based split-and-delay multiplexer (MUX) designed to preserve the $2^{7}-1$ PRBS sequence at the 640-Gbit/s data rate as well as keep the data in one single polarization. For pulse compression, the data pulses are chirped by Self Phase Modulation (SPM) in $400 \mathrm{~m}$ of dispersion flattened highly nonlinear fiber (HNLF). The positive dispersion in the remainder of the transmitter, corresponding to $20 \mathrm{~m} \mathrm{SMF}$, linearly compresses the data pulses to $\sim 530 \mathrm{fs}$ FWHM in the resulting 640-Gbit/s data signal. An eye diagram of the 640-Gbit/s data, obtained with an optical sampling oscilloscope, is also shown in Fig. 1. This shows clear and open and well-equalized 640-Gbit/s data eyes.

The data signal is sent to the receiver with the clock recovery unit and a NOLM-based demultiplexer or it is first guided through a dispersion and slope-compensated fiber span of $50-\mathrm{km}$ SMF-IDF. The total $50-\mathrm{km}$ span residual dispersion is $0.13 \mathrm{ps} / \mathrm{nm}$ and the slope is $0.07 \mathrm{ps} / \mathrm{nm}^{2}$, which is within the general requirements for less than $1-\mathrm{dB}$ transmission penalty for 640-Gbit/s transmission and leads to a pulse broadening of less than $100 \mathrm{fs}$. The PMD in this span is negligible, as there is no noticeable effect of tuning the polarization into the span. In the clock recovery setup, the data signal is injected into the ARW PPLN, which acts as a phase comparator between the data and a local clock signal, thus generating an error signal, proportional to the sine of the phase difference between the clock and data. This error signal is then used to lock the loop to the data signal frequency.

The ARW PPLN used in this experiment, sketched in Fig. 2, is designed for efficient $\chi^{(2)}$ processes from the $1550 \mathrm{~nm}$ wavelength range [13]. Active $\mathrm{Mg}: \mathrm{LiNbO}_{3}$ is set on a low-index

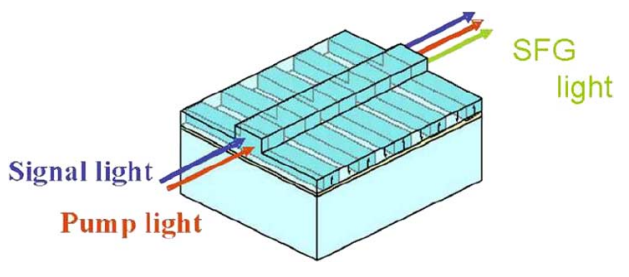

Fig. 2. Schematic of the used PPLN module.

adhesive, and together with a ridge structure this gives an optical waveguide with a tight optical confinement with an index difference of $\Delta \mathrm{n} \sim 0.6$ [13]. The ridge $(2.5 \mu \mathrm{m}$ high and $8 \mu \mathrm{m}$ wide) ensures good modal overlap between light at the fundamental wavelength $(1550 \mathrm{~nm})$ and the second harmonic $(780$ $\mathrm{nm}$ ), which will enhance the conversion efficiency. The mode size at both wavelengths is about $3 \mu \mathrm{m}$. Traditionally $\mathrm{LiNbO}_{3}$ is attractive because of its high $\chi^{(2)}$ with fs-timescale response, but drawbacks are pulse and beam walk-off due to group velocity dispersion (GVD) and birefringence in the crystal, respectively, which both tend to reduce the effective interaction length. Periodic poling greatly reduces the birefringence-induced beam walk-off by periodically changing the sign of $\chi^{(2)}$, making the process quasi-phase-matched (QPM). The GVD-induced pulse walk-off is reduced simply by reducing the length of the device. This is made possible by increasing the normalized conversion efficiency per length, enabled by the ridge structure resulting in good modal overlap between the fundamental and second harmonic. The length of the device is $\sim 30 \mathrm{~mm}$ and the QPM period $17 \mu \mathrm{m}$, resulting in efficient sum-frequency generation (SFG) between the $1557 \mathrm{~nm}$ data and the $1567 \mathrm{~nm}$ clock at $782 \mathrm{~nm}$. The normalized conversion efficiency for the $30 \mathrm{~mm}$ device is $\eta_{\text {conv }} \sim 350 \% / \mathrm{W}$ for the packaged module, and $900 \% / \mathrm{W}$ for the naked chip. The $\chi^{(2)}$ process takes place on an fs-timescale, as mentioned above. However, due to dispersion in the material, the group velocity mismatch (GVM) between the fundamental $1560-\mathrm{nm}$ wave and the second-harmonic $780-\mathrm{nm}$ wave amounts to $0.29 \mathrm{ps} / \mathrm{nm}$, giving $8.7 \mathrm{ps}$ in the packaged module. The clock and data pulses, both in the 1560-nm range, have negligible GVM between them $(0.356 \mathrm{fs} / \mathrm{mm}$ giving $10.7 \mathrm{fs}$ for the module), so this means that for the purpose of clock recovery, the timing resolution is not limited by the module, but by the obtainable pulse widths for the data and clock pulses. Note that due to the GVM between the fundamental and the harmonic, 
the produced error signal will be broadened by the $8.7 \mathrm{ps}$, but this has no consequence for the application here, since the error signal will be varying only very slowly. The GVM only puts a limit on the repetition rate of the clock pulses, not on the data pulses. The clock pulse repetition rate should thus not exceed $1 / 8.7$ ps $\sim 100 \mathrm{GHz}$. Thus, the customary $10-$ or $40-\mathrm{GHz}$ base rate is more than accommodated for. However, the data signal could in principle be extended to $1 \mathrm{Tbit} / \mathrm{s}$. To summarize the design principle, a high normalized conversion efficiency per unit length in the ARW PPLN, obtained by the ridge structure, enables reduced device length, leading to a small group velocity mismatch and a large wavelength bandwidth, making it suitable for ultrafast operation.

The local clock signal consists of $10-\mathrm{GHz}$ pulses from a semiconductor tunable mode-locked laser (TMLL) driven by a voltage-controlled oscillator (VCO), which in turn is tuned by the error signal. The overall bit-rate limitation is set by the pulsewidth of the local clock and the response time of the mixing process, so short pulses and fast mixers are required. The TMLL runs at $1567 \mathrm{~nm}$, fulfilling the phase-matching condition with the 1557-nm data, and pulse compression by soliton compression in a high-power EDFA is employed. The TMLL pulse is amplified to $\sim 30 \mathrm{dBm}$ and injected into a second $30-\mathrm{dBm}$ amplifier where a soliton is excited in the Er-doped fiber, which has a positive gain. When the soliton travels through the Er-fiber, the peak power will increase due to the gain, and hence self-phase modulation (SPM) will dominate over GVD leading to an adiabatic compression. The compressed pulses have a 700-fs FWHM pulse width, and a pedestal at roughly $20 \%$ of the pulse peak power (bottom of Fig. 4), which is expected to reduce the contrast of the error signal. The overall loop length with pigtails and EDFAs is about $60 \mathrm{~m}$, and, with a PLL bandwidth of $200 \mathrm{kHz}$, the loop is expected to be stable, according to [14].

Fig. 3 shows the spectra of the input and output signals to and from the ARW PPLN together with the generated error signal. When the clock and the data pulses overlap in time in the PPLN, a sum-frequency product at $782 \mathrm{~nm}$ will be generated. Based on the measurements shown here, the $\eta_{\text {conv }}$ of the packaged module at $10-\mathrm{mW}$ input power is $-14 \mathrm{~dB} \sim 4 \%$.

Fig. 4 shows a cross-correlation trace of the 640-Gbit/s data signal with a 500-fs sampling pulse, confirming that the data signal is of good quality as indicated by the eye diagrams in Fig. 1 . The individual channels are correctly separated by 1.57 ps, and they are well-equalized. The same transmitter is used in [15], where it is shown that all channels are error-free with about $3-\mathrm{dB}$ variation in sensitivity.

Fig. 4 (bottom) shows an autocorrelation trace of the local clock pulses, with the clearly visible pulse pedestal mentioned above. This pedestal is expected to reduce the contrast of the error signal, but not limit the clock recovery performance.

If the clock and data signals are not synchronised they will scan across each other at the difference frequency, generating a slowly varying error signal, see Fig. 5. The error signal at 320 Gbit/s clearly resolves the full data signal, even showing the plateaus between the pulses. This signifies that the SFG-process in this component is ultrafast, as this trace includes the effects of the design of the module, including length. The contrast of the

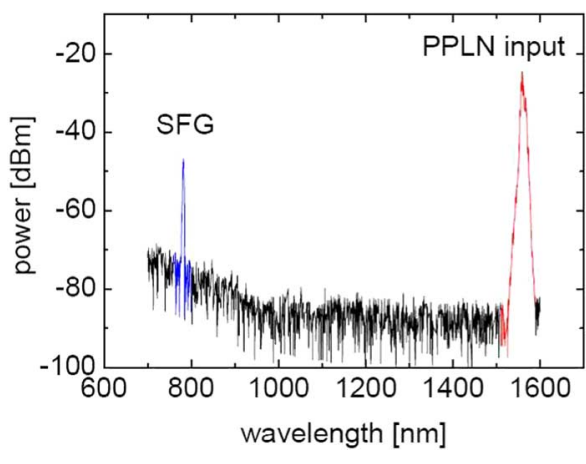

(a)

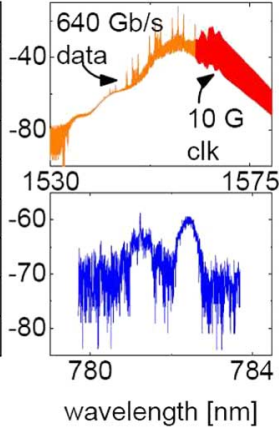

(b)
Fig. 3. (a) PPLN optical spectral output, showing the clock-data around 1550 $\mathrm{nm}$ and the SFG output at $782 \mathrm{~nm}$. (b) Zoom in on 640-Gbit/s input spectrum overlapping with $10-\mathrm{GHz}$ input clock (top) and zoom in on the SFG output of the PPLN at $780 \mathrm{~nm}$ (bottom).
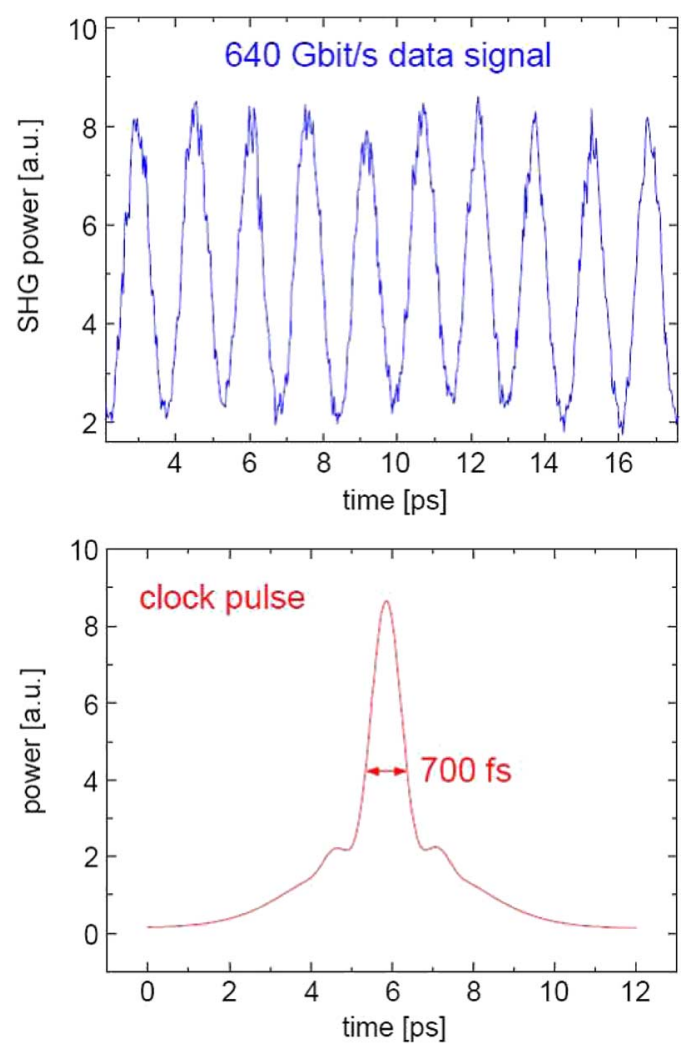

Fig. 4. Clock and data pulses. Top: cross correlation. Of the 640-Gbit/s data signal with a 500-fs sampling pulse, showing a good quality signal with wellequalized individual channels, corroborating the 640-Gbit/s eye diagram inset in Fig. 1. Bottom: auto-correlation of the $10-\mathrm{GHz}$ clock pulse with $20 \%$ pedestal.

$320-\mathrm{Gbit} / \mathrm{s}$ data signal is approximately $20 \%$, which is expected to be due to the clock pulse pedestals and some background second-harmonic generation.

The 640-Gbit/s error signal also clearly resolves the individual data pulses, although with a reduced contrast (about $10 \%$ ), primarily owing to the width of and pedestals on the clock pulses. The error signals are perfectly suitable for locking, and successful clock recovery at both 320 and $640 \mathrm{Gbit} / \mathrm{s}$ is achieved. Please note that the polarization needs to be set very 


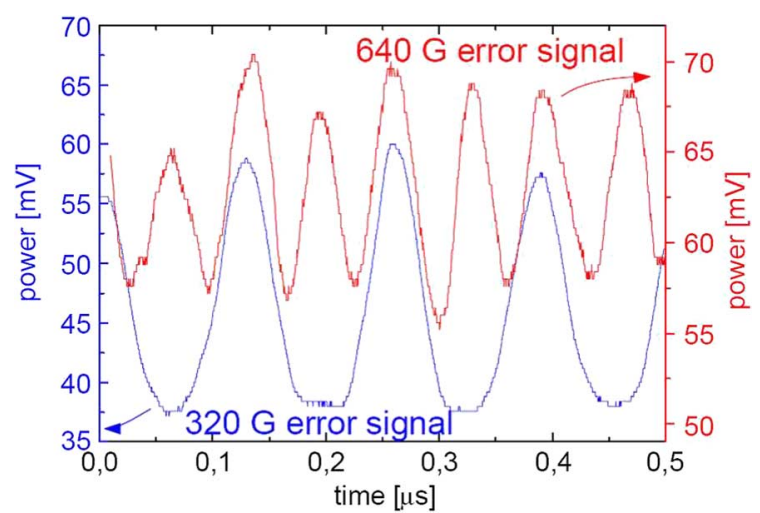

Fig. 5. Generated error signals at 320 and $640 \mathrm{Gbit} / \mathrm{s}$ showing a good temporal resolution and a sufficient contrast.

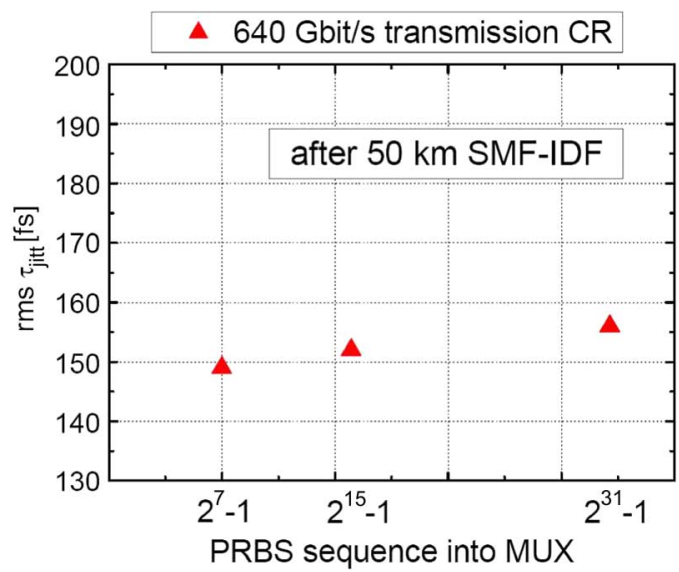

Fig. 6. RMS timing jitter for various input PRBS sequences-less than $10 \mathrm{fs}$ difference is observed.

carefully on both the data and the clock pulses when they enter the waveguide, to achieve phase matching.

\section{EXPERIMENTAL RESULTS}

The produced error signals at 320 and $640 \mathrm{Gbit} / \mathrm{s}$ are used successfully for clock recovery both before and after transmission. To make the situation as realistic as possible, most of the characterization measurements presented in the following are performed after transmission.

Fig. 6 shows the 640-Gbit/s locking performance after 50-km transmission in terms of the integrated timing jitter derived from the single-sideband-to-carrier ratio (SSCR) phase-noise (integration range: $1 \mathrm{kHz}-1 \mathrm{GHz}$ ). Fig. 6 shows the timing jitter directly out of the VCO when locking to the 640-Gbit/s transmitted data, showing around 150 -fs rms timing jitter.

Since SFG in the PPLN is almost instantaneous, the clock recovery is expected to be independent of the OOK data pattern it receives. To investigate this, the PRBS sequence into the multiplexer is changed. The multiplexer is PRBS-maintaining only for a $2^{7}-1$ word length, though, so in this characterization the PPLN does not receive pure PRBS sequences for the higher word lengths. However, the bit sequences still remain very different to each other for different input sequences. The rms timing jitter is around $150 \mathrm{fs}$, and this number only changes

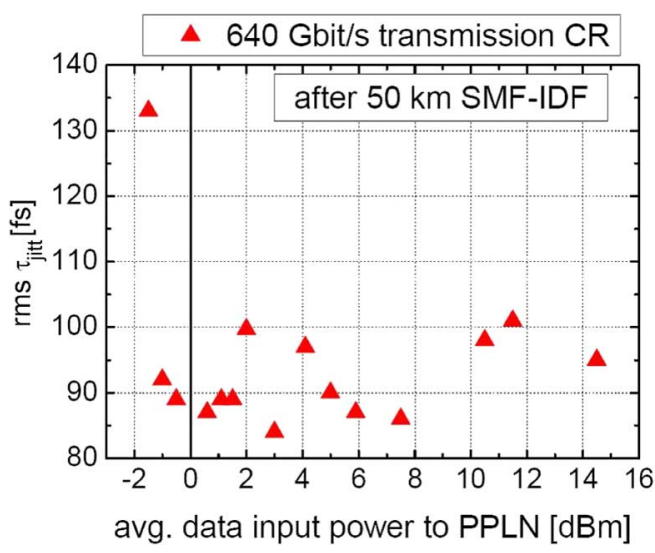

Fig. 7. Timing jitter for various average data input powers-less than 100-fs jitter is obtained for a dynamic range of more then $15 \mathrm{~dB} ; 1 \mathrm{~mW}$ data power is sufficient.

by 10 fs when changing the PRBS sequence input to the multiplexer in the transmitter, effectively confirming the theoretical expectation of no pattern dependence. So, no pattern dependence is expected, and within the limits of this setup, none are observed.

When locked, the clock recovery is locked on one of the 64 tributaries, and even though the aggregate 640-Gbit/s signal is not a $2^{31}-1$ PRBS, each tributary is, and thus this investigation shows that the PLL can lock to long sequences of zeroes.

The PLL is designed to have a hold-in range of $37.5 \mathrm{MHz}$, i.e., it can tolerate the data frequency to drift that much and still maintain locking. The pull-in range of the PLL is $8.8 \mathrm{MHz}$, so the VCO and data frequency offset should not exceed this, if the PLL is expected to pull into locking.

The rms timing jitter values in Fig. 6 are directly out of the VCO, but this is not what is fed to the demultiplexer. The VCO controls an ERGO laser, which in turn controls the demultiplexer. The ERGO laser itself has very low jitter and a quite low PLL bandwidth of $20 \mathrm{kHz}$. This means, in effect, that the noise above $20 \mathrm{kHz}$ will be filtered away yielding lower timing jitter on the actual control pulses. Fig. 7 shows the rms timing jitter from the control pulse source applied to the NOLM demultiplexer for various average data input powers, when the average clock power is $-4 \mathrm{dBm}$. The ERGO filters away phase noise from the $\mathrm{VCO}$ above $20 \mathrm{kHz}$, and hence this timing jitter is somewhat lower than the values straight out of the VCO in Fig. 6. This helps in getting error-free demultiplexing as shown in Fig. 8.

Fig. 7 shows that less than 100 fs jitter can be obtained for this system, for average data input powers ranging from -1 to $+15 \mathrm{dBm}$, giving an experimentally obtained dynamic range of $16 \mathrm{~dB}$. This means that $1-\mathrm{mW}$ average data power is enough for this scheme to work. When going below $-1 \mathrm{dBm}$ input power, the signal-to-noise ratio (SNR) out of the detector simply gets too low. For higher data input powers, the pump will eventually get depleted, and there will be more SHG from the data itself giving rise to a bigger offset in the error signal, inevitably leading to loss of locking. Where this occurs cannot be quantified with the present setup, since there is not enough power available. 


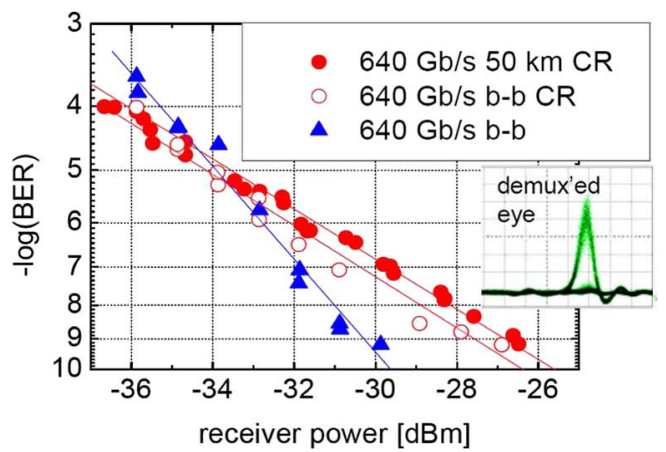

Fig. 8. BER curves for $640-\mathrm{Gbit} / \mathrm{s}$ demultiplexing to $10 \mathrm{Gbit} / \mathrm{s}$ with the clock derived from the clock recovery before and after transmission compared with the $640 \mathrm{Gbit} / \mathrm{s}$ back-to-back. There is an only $\sim 2.7 \mathrm{~dB} \mathrm{CR}$ penalty and less than $1 \mathrm{~dB}$ transmission penalty. Insert: demultiplexed eye after $50-\mathrm{km}$ transmission.

Fig. 8 shows the BER results when using the recovered clock to drive the control pulse source for the NOLM demultiplexer. The receiver power is measured after the multiplexer, just before entering the pre-amplifier receiver. The 640-Gbit/s back-toback (b-b) demultiplexing is error free (i.e., BER $>10^{-9}$ ) with no error floor and has a sensitivity (i.e., receiver power at BER $=10^{-9}$ ) of $-30.3 \mathrm{dBm}$. Compared with the eye diagram in Fig. 1, which seems to show some intersymbol interference (ISI), there is no such sign in the BER curves. This is because there is no ISI, as the eye diagram interference is an artefact of the sampling oscilloscope used. It uses a 900-fs sampling pulse, and this will overlap with neighboring channels, giving the appearance of ISI. When comparing to the cross-correlation traces in Fig. 4, which uses a 500-fs sampling pulse and thus has a higher temporal resolution than the sampling oscilloscope, it is verified that there is no noticeable pulse overlap between channels. Using the CR without transmission (i.e., b-b), error-free performance is readily achieved with a sensitivity of $-27.6 \mathrm{dBm}$, i.e., a penalty of only $2.7 \mathrm{~dB}$. Using the $\mathrm{CR}$ after transmission is also successful and error-free performance is achieved, with an additional penalty of only $0.8 \mathrm{~dB}$. These results clearly demonstrate that the PPLN module works satisfactorily in the full $640-\mathrm{Gbit} / \mathrm{s}$ transmission system. Please note that the b-b 640-Gbit/s test-bed is very stable and all 64 channels are error-free with a sensitivity spread of $3.3 \mathrm{~dB}$, as more thoroughly described in [15]. The channels shown in Fig. 8 are typical channels taken from the middle of the 3.3-dB spread, as verified by scanning through a couple of channels and finding similar sensitivities. This is further corroborated by inspecting the cross-correlation traces of the $640-\mathrm{Gbit} / \mathrm{s}$ data signals, confirming that the channels are still narrow, equally spaced and well-equalized, like the original 640-Gbit/s data. After transmission, the pulse broadening is less than $100 \mathrm{fs}$, effectively rendering the transmitted data signal very similar to the original one.

In order to characterize further the requirement on timing jitter to obtain a BER $<10^{-9}$, the BER corresponding to different phase noise curves is measured.

Fig. 9 shows characterization results of the phase noise and derived timing jitter after transmission of the $640-\mathrm{Gbit} / \mathrm{s}$ data. The recovered clock signal straight out of the VCO is compared to the pulses out of the ERGO locked to the VCO. As seen in
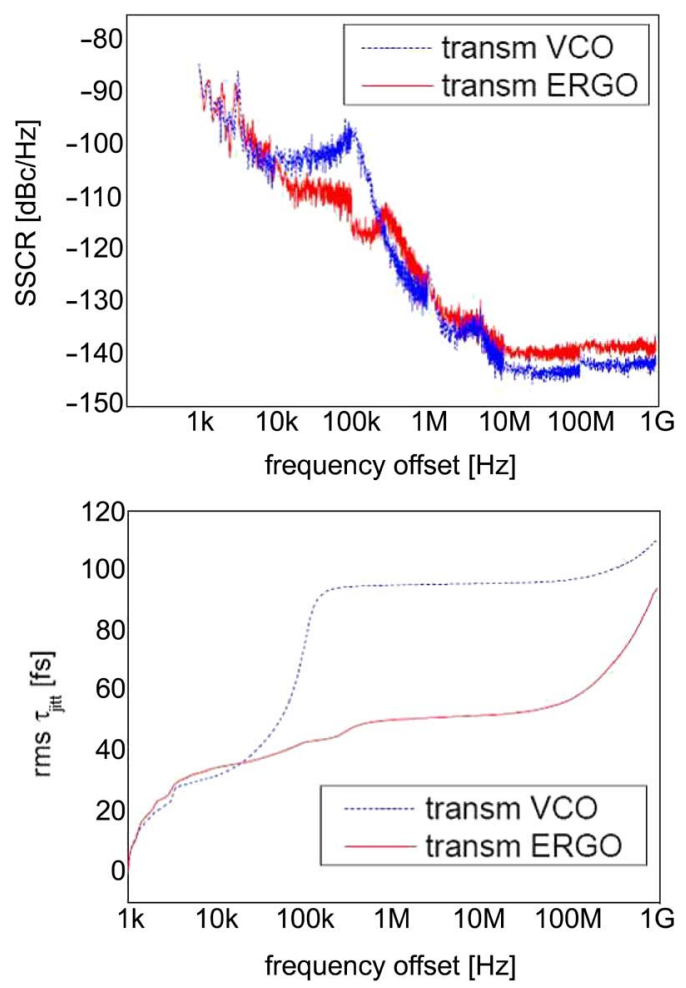

Fig. 9. Jitter filtering by the control pulse source. Top: SSCR of the recovered clock for the 640-Gbit/s transmitted data straight out of the VCO and after the ERGO laser. Bottom: Integrated rms timing jitter as a function of the upper integration limit (integration from $1 \mathrm{kHz}$ and upwards) for the $\mathrm{VCO}$ and the ERGO after transmission of the 640-Gbit/s data.

Fig. 9 (top), the ERGO cuts away excess phase noise above the ERGO PLL bandwidth of $\sim 20 \mathrm{kHz}$. In the VCO SSCR, there is a peak at about $200 \mathrm{kHz}$, which stems from the PLL bandwidth of about $200 \mathrm{kHz}$, It is at this frequency that the PLL shifts from tracking the data SSCR to following the VCO SSCR [14]. This peak and the phase noise associated with it is eliminated by the ERGO.

Fig. 9 (bottom) shows the integrated rms timing jitter values with the lower integration range fixed at $1 \mathrm{kHz}$ and as a function of the upper integration limit. This plot enables one to see in which frequency range the phase noise gives rise to most timing jitter. A big difference is observed between the VCO and the ERGO phase noise and jitter. While the VCO jitter continues to increase to more than $100 \mathrm{fs}$ beyond the $20 \mathrm{kHz}$ point, the ERGO jitter remains well below $100 \mathrm{fs}$. This implies that below $20 \mathrm{kHz}$, the VCO noise will have a direct impact on the demultiplexing, but the VCO noise above $20 \mathrm{kHz}$ is less important, as long as it is clean enough for the ERGO to be able to lock to it. According to the rule of thumb provided in [16], the timing jitter for the control pulse for $640 \mathrm{Gbit} / \mathrm{s}$ should be around $90 \mathrm{fs}$, which can be obtained with this scheme. To quantify this rule of thumb, the BER is measured.

Fig. 10 displays the relation between BER and VCO timing jitter and the SSCR at $10-\mathrm{kHz}$ offset from the carrier, as this will be transferred to the ERGO. Fig. 10 (top) shows the SSCR spectrum for two cases: one for low integrated jitter (150 fs) and one for high jitter (170 fs). The biggest difference between the two traces is around and below $10 \mathrm{kHz}$ where there is about 

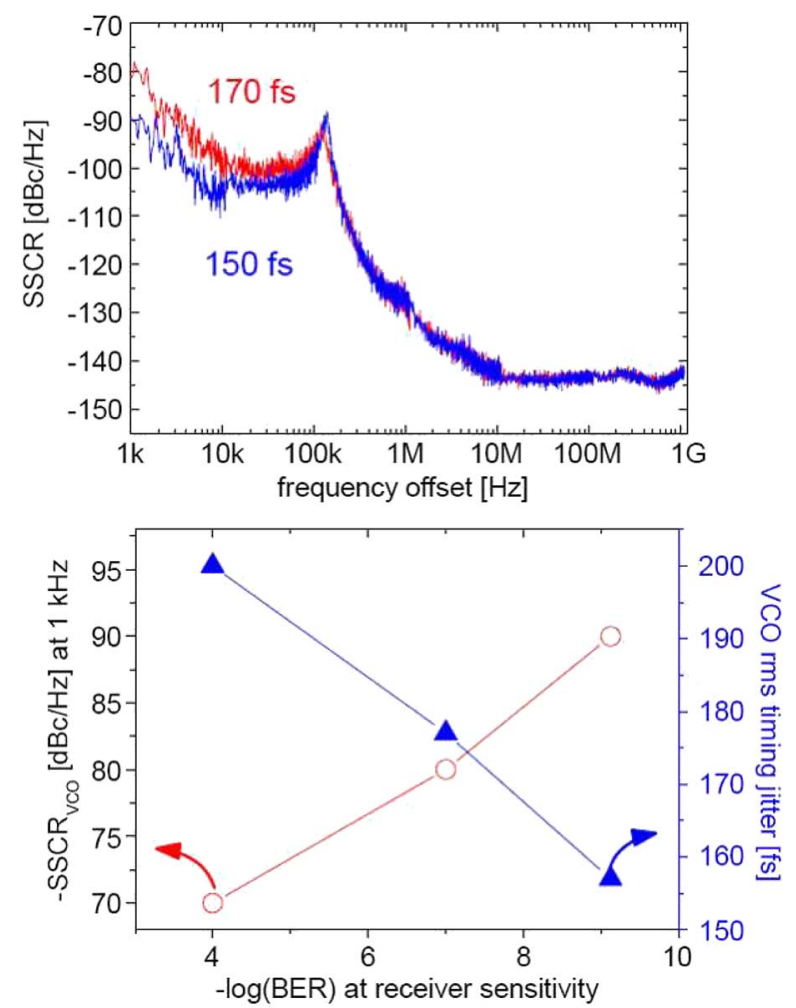

Fig. 10. Relation between $\mathrm{SSCR}[\mathrm{dBc} / \mathrm{Hz}]$ at $10 \mathrm{kHz}$ from the carrier, $\mathrm{VCO}$ timing jitter, and the BER of the demultiplexed signal. Top: SSCR for a low and a high timing jitter VCO. Bottom: connection between BER, SSCR, and rms timing jitter.

$10 \mathrm{dBc} / \mathrm{Hz}$ difference. Comparing such SSCR traces to the obtained BER values at the demultiplexer output gives the link between BER and rms timing jitter (see the bottom of Fig. 10). As observed, there is a huge difference in BER performance when changing the SSCR $10 \mathrm{dBc} / \mathrm{Hz}$ in the $10-\mathrm{kHz}$ range. Demultiplexing only becomes error-free with VCO jitter below 160 fs, and this in turn corresponds to less than 100 -fs jitter on the ERGO. It is thus experimentally found (for this setup) that a $|\mathrm{SSCR}|>90 \mathrm{dBc} / \mathrm{Hz}$ is required on the VCO output at $10-\mathrm{kHz}$ offset, corresponding to an rms jitter of less than $160 \mathrm{fs}$, to obtain error-free demultiplexing. The control ERGO laser then filters away the rest excess phase noise to get below $100 \mathrm{fs}$, and this result therefore agrees well with the rule of thumb in [16]. As shown in Fig. 7, this setup can readily provide less than 100 -fs timing jitter in a large dynamic range. Please note that with the pulse compression techniques used here, it is empirically found that, under optimum working conditions, only about 10 -fs timing jitter is added from the compression stage.

\section{DISCUSSION}

The timing jitter values obtained here are quite good considering the large phase-noise contribution from the TMLL, which has a free-running rms timing jitter of $\sim 400 \mathrm{fs}$. The reason for the low jitter values is a very low-noise VCO and the low bandwidth of the PLL. Replacing the TMLL with a low-jitter laser as in [17], the overall jitter obtained in this setup is expected to become even lower or more stably so.

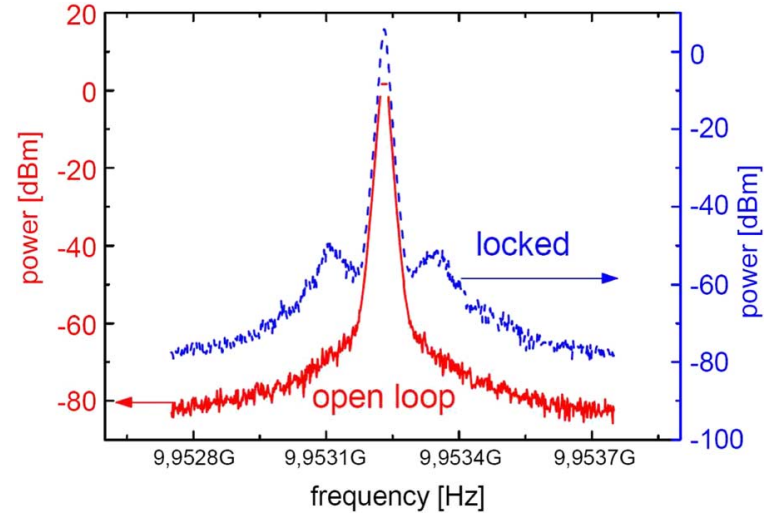

Fig. 11. RF spectra of the free-running VCO (open-loop) and the locked VCO (locked).

Fig. 11 shows the RF electrical power spectra of the freerunning (open loop) VCO and the closed-loop locked VCO. The free-running VCO has a very clean carrier peak with very low noise around it ( $60 \mathrm{~dB} \mathrm{SNR})$. When the loop is closed, however, the noise from the TMLL is circulated around in the loop and this is added to the locked VCO spectrum. The $200-\mathrm{kHz}$ peaks, corresponding to the PLL bandwidth, is also clearly observed here. Within $200 \mathrm{kHz}$, the VCO tracks the data, and beyond the VCO follows its own noise (plus the noise added from the TMLL). The SNR in this case is about $50 \mathrm{~dB}$, i.e., which is clearly lower than the VCO's own noise. This again leads to the interpretation that a laser with lower noise will improve the performance.

Reducing the loop length from its present $\sim 60 \mathrm{~m}$, allowing for an expansion of the PLL bandwidth, will also help to lower the timing jitter, as the influence of the low-jitter data signal in this setup will dominate [14].

It is worth noting that using flat-top switching windows, as demonstrated in [18], timing jitter up to $22 \%$ of the timeslot can be tolerated, which for $640 \mathrm{Gbit} / \mathrm{s}$ corresponds to $350 \mathrm{fs}$. In that case, the requirements on the presented scheme here would be greatly relaxed and should make this whole scheme even more stable than the present version.

Regarding stability, if practically implementing this scheme in a real transmission system, the inherent polarization dependence of the PPLN will need to be addressed. There has already been demonstrated various polarization diversity schemes for other polarization-dependent switches, and we would speculate that these would also be applicable here. For instance, one could add a polarization beam-splitter (PBS) in front of the PPLN and then apply a half-wave plate in one arm before merging the two PBS outputs and injecting the signals into the PPLN. This should alleviate the polarization influence on the switch, but would need further investigations. Apart from that, the switch is already very compact and stable, being packaged into a fiber-pigtailed temperature stabilized module.

In the demonstration here, the tolerance to transmission span parameters has not been directly investigated. However, there are some general requirements on dispersion for getting less 
than $1-\mathrm{dB}$ power penalty at a BER $=10^{-9}$, as stated in Section II [19]. These requirements are more to do with the demultiplexing, but there are also limitations on the clock recovery. If the data pulses get too broad, the error signal will become too small, and locking will not be possible. The requirement will be slightly less stringent than for demultiplexing, but on the same order of magnitude, as the data pulses still need to be sufficiently narrow to be properly distinguishable in the PPLN.

\section{CONCLUSION}

We have reported on a novel clock recovery scheme, relying on truly ultrafast sum-frequency generation in an ARW PPLN. The temporal resolution of the setup was sufficient to resolve a 640-Gbit/s OTDM data signal, and locking at bit rates up to 640 Gbit/s was successfully achieved before and after transmission over $50 \mathrm{~km}$ of SMF-IDF fiber. Timing jitter of less than $100 \mathrm{fs}$ was obtained for this system with a dynamic range of $16 \mathrm{~dB}$. No pattern dependence was expected and none was found. The clock recovery unit gave error-free performance with excellent quality and less than $1-\mathrm{dB}$ transmission penalty. Only $0 \mathrm{dBm}$ average power in the 640-Gbit/s data signal was needed and only $-4 \mathrm{dBm}$ clock power was used, so this is a low-power solution for clock recovery. The overall power usage in this proof-ofprinciple laboratory implementation for the full receiver is, however, not particularly low-power, as we here need to use various tricks for pulse compression and need about $20 \mathrm{dBm}$ control power to the NOLM. In a future setup, quantum-dot modelocked lasers have been shown to generate sub-ps pulses with very low timing jitter, so pulse compression could be avoided. The results presented in this paper constitute the first demonstration of the use of a PPLN at such high bit rates and is only the second demonstration of 640-Gbit/s clock recovery and the second full (i.e., including line rate clock recovery) 640-Gbit/s transmission demonstration ever.

\section{REFERENCES}

[1] A. H. Gnauck, G. Charlet, P. Tran, P. Winzer, C. Doerr, J. Centanni, E. Burrows, T. Kawanishi, T. Sakamoto, and K. Higuma, "25.6-Tb/s C+L-band transmission of polarization-multiplexed RZ-DQPSK signals," presented at the Proc. Optical Fiber Commun. Conf., 2007, postdeadline paper PDP19, unpublished.

[2] B. Metcalfe [Online]. Available: http://www.ofcnfoec.org/conference_program/Plenary.aspx

[3] K. Schuh, B. Junginger, E. Lach, and G. Veith, " $1 \mathrm{Tbit} / \mathrm{s}(10 \times 107$ Gbit/s ETDM) NRZ transmission over $480 \mathrm{~km}$ SSMF," presented at the Proc. Optical Fiber Commun. Conf., 2007, postdeadline paper PDP23, unpublished.

[4] M. Nakazawa, T. Yamamoto, and K. R. Tamura, "1.28 Tbit/s-70 km OTDM transmission using third- and fourth-order simultaneous dispersion compensation with a phase modulator," Electron. Lett., vol. 36, no. 24, pp. 2027-2029, 2000.

[5] H. G. Weber, C. Schubert, S. Ferber, M. Kroh, C. Schmidt-Langhorst, R. Ludwig, V. Marembert, C. Boerner, F. Futami, and S. Watanabe, "Single channel $1.28 \mathrm{Tbit} / \mathrm{s}$ and $2.56 \mathrm{Tbit} / \mathrm{s}$ DQPSK transmission," Electron. Lett., vol. 42, no. 3, pp. 178-179, 2006.

[6] E. Tangdiongga, H. C. H. Mulvad, H. de Waardt, G. D. Khoe, A. M. J. Koonen, and H. J. S. Dorren, "SOA-based clock recovery and demultiplexing in a lab trial of $640 \mathrm{~Gb} / \mathrm{s}$ OTDM transmission over $50-\mathrm{km}$ fibre link," presented at the Proc. Eur. Conf. Optical Commun., 2007, Postdeadline paper PD 1.2, unpublished.
[7] O. Kamatani and S. Kawanishi, "Prescaled timing extraction from 400 $\mathrm{Gb} / \mathrm{s}$ optical signal using a phase lock loop based on four-wave-mixing in a laser diode amplifier," IEEE Photon. Technol. Lett., vol. 8, no. 8, pp. 1094-1096, Aug. 1996.

[8] L. K. Oxenløwe, D. Zibar, M. Galili, A. T. Clausen, L. J. Christiansen, and P. Jeppesen, "Filtering-assisted cross-phase modulation in a semiconductor optical amplifier enabling $320 \mathrm{~Gb} / \mathrm{s}$ clock recovery," presented at the Proc. Eur. Conf. Optical Commun., Glasgow, U.K., 2005, paper We3.5.5, vol. 3, pp. 485-487, unpublished.

[9] H. C. H. Mulvad, E. Tangdiongga, H. de Waardt, and H. J. S. Dorren, "40 GHz clock recovery from $640 \mathrm{Gbit} / \mathrm{s}$ OTDM signal using SOA based phase comparator," Electron. Lett., vol. 44, no. 2, pp. 146-147, Jan. 2008.

[10] F. Gómez Agis, C. Ware, D. Erasme, R. Ricken, V. Quiring, and W. Sohler, "10-GHz clock recovery using an optoelectronic phase-locked loop based on three-wave mixing in periodically poled lithium niobate," IEEE Photon. Technol. Lett., vol. 18, no. 13, pp. 1460-1462, Jul. 2006.

[11] C. Langrock, S. Kumar, J. E. McGeehan, and M. M. Fejer, "All-optical signal processing using-(2) nonlinearities in guided-wave devices," $J$. Lightwave Technol., vol. 26, no. 7, pp. 2579-2592, Jul. 2006.

[12] J. E. McGeehan, M. Giltrelli, and A. E. Willner, "All-optical digital 3 -input and gate using sum- and difference-frequency generation in a PPLN waveguide," in Dig. LEOS Summer Top. Meetings, Munich, Germany, Jul. 2005, pp. 179-180.

[13] S. Kurimura, Y. Kato, M. Maruyama, Y. Usui, and H. Nakajima, "Quasi-phase-matched adhered ridge waveguide in LiNbO3," Appl. Phys. Lett., vol. 89, p. 191123, 2006.

[14] D. Zibar, J. Mørk, L. K. Oxenløwe, and A. T. Clausen, "Phase noise analysis of clock recovery based on an optoelectronic phase-locked loop," J. Lightw. Technol., vol. 25, no. 3, pp. 901-914, Mar. 2007.

[15] M. Galili, H. C. H. Mulvad, L. K. Oxenløwe, A. T. Clausen, and P. Jeppesen, "Optical wavelength conversion by cross-phase modulation of data signals up to $640 \mathrm{~Gb} / \mathrm{s}$," IEEE J. Sel. Topics Quantum Electron, vol. 14, no. 3, pp. 573-579, May-Jun. 2008.

[16] M. Jinno, "Effects of crosstalk and timing jitter on all-optical timedivision demultiplexing using a nonlinear fiber sagnac interferometer switch," IEEE J. Quantum Electron, vol. 30, no. 12, Dec. 1994.

[17] K. Yvind, D. Larsson, L. J. Christiansen, L. K. Oxenløwe, J. Mørk, J. M. Hvam, and J. Hanberg, "Design and evaluation of mode-locked semiconductor lasers for low noise and high stability (invited)," in Proc. OptoIreland Conf., 2005.

[18] L. K. Oxenløwe, R. Slavík, M. Galili, H. C. H. Mulvad, A. T. Clausen, Y. Park, J. Azaña, and P. Jeppesen, " $640 \mathrm{~Gb} / \mathrm{s}$ timing jitter-tolerant data processing using a long-period fiber-grating-based flat-top pulse shaper," IEEE J. Sel. Topics Quantum Electron, vol. 14, no. 3, May-Jun. 2008.

[19] A. T. Clausen, H. N. Poulsen, L. Oxenløwe, A. I. Siahlo, J. Seoane, and P. Jeppesen, "Pulse source requirements for OTDM systems," presented at the IEEE Laser and Electro-Optics Soc. Annu. Meeting, Tucson, AZ, 2003, paper TuY2, unpublished.

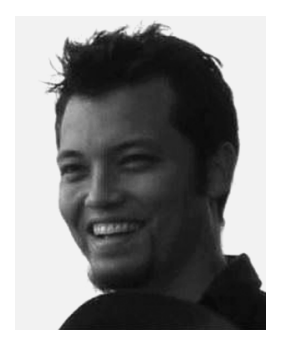

Leif Katsuo Oxenløwe received the B.Sc. degree in physics and astronomy from the Niels Bohr Institute, University of Copenhagen, Copenhagen, Denmark, in 1996, the International Diploma of Imperial College of Science, Technology and Medicine, London, U.K., in 1998, the M.Sc. degree from the University of Copenhagen, in 1998, and the Ph.D. degree from the Technical University of Denmark, Lyngby, in 2002.

He is currently an Associate Professor with DTU Fotonik, Department of Photonics Engineering, Technical University of Denmark, Lyngby, where he is the group leader of the Ultra-high-speed Optical Communications Group. He is working with experimental research in the field of ultrafast optical communications (above $160 \mathrm{~Gb} / \mathrm{s})$. He has been working within the EU IST project TOPRATE and the Danish Research Council-financed project SCOOP. Starting in May 2004, he joined and managed the project ULTRA-NET funded by the Danish research council. In August 2007, he embarked on the project NANO-COM also funded by the Danish Research Council, and this project deals with nanotechnology-based solutions for ultrahigh-bit-rate communications. He has authored or coauthored more than 110 peer-reviewed publications. 


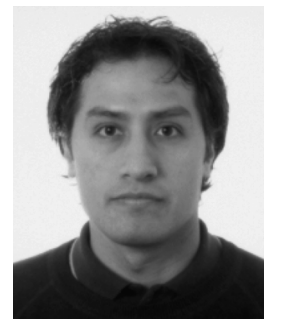

Fausto Gómez-Agis received the B.Sc. degree in electronics from the Instituto Tecnológico de Mazatlán, Sinaloa, México, in 1996 and the M.Sc. degree in optics from CICESE, Ensenada, B.C., México, in 1999. He is currently working toward the Ph.D. degree at École Nationale Supérieure des Télécommunications (now TELECOM ParisTech), Paris, France.

In 2000, he joined the Last-Mile Access Department of TELMEX (Teléfonos de México), Jalisco, México, as a Systems Engineer. From 2001 to 2004, he was with CIDEC (CONDUMEX R\&D center), Querétaro, México, where he was involved with research activities on plastic optical fiber communications such as design, construction and evaluation of devices for Fast-Ethernet LAN applications. He is currently involved in research on optoelectronic clock recovery.

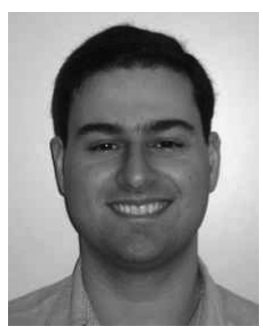

Cédric Ware was born in Paris, France, on January 24, 1977. He received the B.Sc. degree in computer science from the École Normale Supérieure, Paris, the M.Sc. degree in physics from the Université Paris 6 and 7, Paris, in 1996, and the Engineering and Ph.D. degrees from the École Nationale Supérieure des Télécommunications, Paris, in 1998 and 2003, respectively. His doctoral work specialized in optoelectronic clock recovery.

Since 1998, he has been with the Department of Communications and Electronics, École Nationale Supérieure des Télécommunications (now TELECOM ParisTech), as an Assistant Professor. He has been an Associate Professor since 2007. His current research activities include optical signal processing for use in optical communications networks, notably clock recovery, packet label recognition, and optical code-division multiple access. He is a national delegate for the European Cooperation in the Field of Scientific and Technical Research (COST) 288 on nanoscale and ultrafast photonics and the Vice Chairman of its working group on photonics devices. He also participates in COST 291 on digital optical networks and the European Networks of Excellence BONE and EuroFOS.

Dr. Ware is a member of the Optical Society of America.

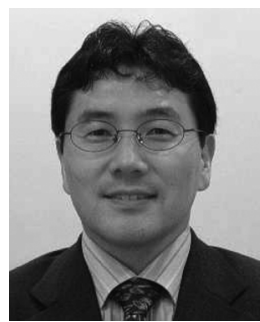

Sunao Kurimura received the Ph.D. degree in engineering from Waseda University, Tokyo, Japan, in 1997.

He was a Visiting Scholar with Stanford University from 1997 to 1999 , and joined the Institute for Molecular Science in Japan as a Research Associate in 1999. He is currently a Principal Researcher with the National Institute for Materials Science, Tsukuba, Japan and has concurrent positions as an Associate Professor with Waseda University and Kyushu University. His current research interests are focused on nonlinear-optical materials and devices with polarity-reversed structures for quasi-phase-matching.

Dr. Kurimura has been a committee member of Conference on Lasers and Electro-Optics since 2006, and will be a subcommittee chair for CLEO 2009. He was the recipient of the Funai Information Science Promotion Award in 2008 for his contribution to quasi-phase-matching nonlinear optics.

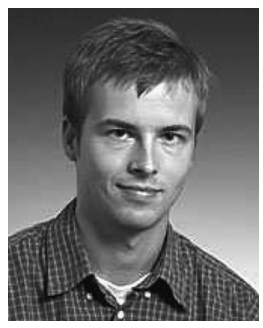

Hans Christian H. Mulvad received the M.Sc. degree in physics from the University of Copenhagen, Copenhagen, Denmark, in 2004. He is currently working towards the Ph.D. degree at DTU Fotonik, Department of Photonics Engineering, Technical University of Denmark, Lyngby.

$\mathrm{He}$ is currently involved in research on fiber nonlinearities for high-speed signal processing. His doctoral work has been focused on creating stable fiberbased switches and switches for 640-Gbit/s add/drop multiplexing.

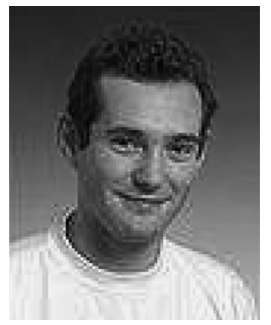

Michael Galili was born in Aabenraa, Denmark, in 1977. He received the M.Eng. degree in applied physics from the Technical University of Denmark, Lyngby, in 2003 and the Ph.D. degree in optical communications and signal processing from the COM Department, Technical University of Denmark (now DTU Fotonik) in 2007. The main focus of his doctoral work was on optical signal processing of high-speed optical data signals.

He joined the Technical University of Denmark as a Ph.D. student in 2004 and is currently a Post Doctoral Fellow. He is the first author or coauthor of more than 30 peer-reviewed scientific publications.

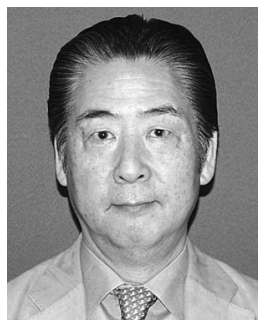

Hirochika Nakajima received B. E., M. E., and Dr. Eng. degrees from Waseda University, Tokyo, Japan, in 1970,1972 , and 1995 , respectively.

He joined Fujitsu Laboratories, Ltd., in 1972, engaging in the research and development of magnetic bubble memory and optical devices. He joined Waseda University as a full Professor of applied physics in 1996. He was a Visiting Professor with Boston University in 1998. He was also the first Japanese "rotator" as a Senior Fellow with the Center for R\&D Strategy (CRDS) of the Japan Science and Technology Agency (JST) during 2003-2006. Since coming back to Waseda University, he has been a Director for Research Promotion.

Dr. Nakajima is a Fellow of the Japan Society of Applied Physics (JSAP) and a member of the Optical Society of America. He was the recipient of the 9th K. Sakurai Memorial Award for his contribution to stabilization of Ti:LiNbO3 waveguide devices in 1993. He occupied a steering committee chair of Microoptics Group under OSJ/JSAP since 2000.

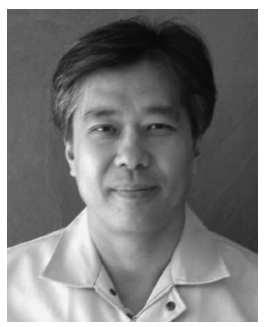

Junichiro Ichikawa received the B.S. and M.S. degrees in mineralogy from the University of Tokyo, Tokyo, Japan, in 1987 and 1989, respectively.

He joined the Optoelectronics Division, Sumitomo Osaka Cement Company, Ltd., Chiba, Japan, in 1989 and has been engaged in development of optical modulators for communication system. He is the first author or coauthor of more than 30 peer-reviewed scientific publications. His recent research interests are in micro-fabrication process of ferroelectric materials for optoelectronics and electronics applications.

Mr. Ichikawa was the recipient of the Sakurai Memorial Award from the Optoelectronic Industry and Technology Development Association (OITDA) of Japan in 2007 for the development on integrated lithium-niobate optical modulators.

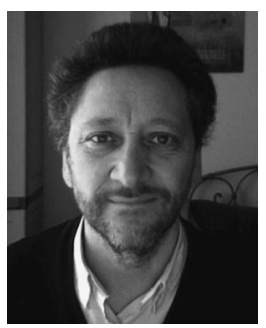

Didier Erasme was born in Paris, France, in 1960. He received the "diplôme d'Ingénieur" in physical engineering from the Ecole Nationale Supérieure d'Ingénieurs Electriciens de Grenoble (INPG), Grenoble, France, in 1983, the Ph.D. degree on LiNbO3 high-frequency integrated-optic modulators from University College London (UCL), London, U.K., in 1987, and the "Habilitation à diriger des recherches" from the Ecole Nationale Supérieure des Télécommunications (Télécom ParisTech), Paris, in 1995.

After two years of postdoctoral work on electrooptic sampling of GaAs integrated circuits at UCL, he joined the Ecole Nationale Supérieure des Télécommunications (Télécom ParisTech) in 1990 as an Associate Professor of optoelectronics. He spent a six-month sabbatical with Prof. M. Smit's group at Delft University, Delft, The Netherlands. He has been a full Professor since 1998. His current research interests are in the area of new optical functions for telecommunication optical systems and networks. Particularly, he has developed a strong interest in semiconductor laser amplifiers and other nonlinear optical devices for applications in all-optical signal processing. Applications range from ultra high-speed clock recovery and OTDM demultiplexing to the realization of sub- 
systems dedicated to optical packet switching nodes. He is the author or coauthor of more than 70 publications and communications in international journals and conferences. He has participated in the European programs OPTIMIST and BREAD dedicated to the road-mapping of European Broadband-for-all activities in Europe and to the European Network of Excellence ePhoton/ONe and now BONE and EUROFOS.

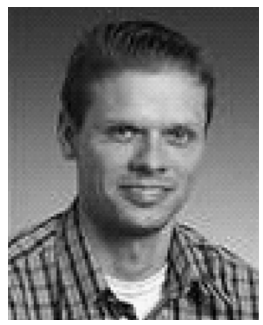

Anders Thomas Clausen was born in Copenhagen, Denmark, in 1967. He received the M.Sc. degree in electrical engineering from the former Electromagnetics Institute (EMI), Technical University of Denmark (DTU), Lyngby, in 1997 and the Ph.D. degree from DTU Fotonik, DTU, in 2007.

$\mathrm{He}$ carried out his military service during 1995-1996. He joined EMI as a Research Associate in 1997, working on optical signal processing. He was participating in a number of European projects, such as HIGHWAY, REPEAT and METEOR and national projects. In 2001, he became group leader of an Optical Time-Division Multiplexing (OTDM) group at COM. The OTDM group was heavily engaged in the European project TOPRATE. His doctoral work at COM focused on experimental and theoretical investigations of systems with potential for terabit capacity. He is now a Post-Doctoral Fellow with DTU Fotonik. He is the first author or coauthor of more than 100 peer-reviewed scientific publications. His research interests are primarily focused on ultrahigh-speed signal processing at bit rates of $160 \mathrm{Gbit} / \mathrm{s}$ and beyond.

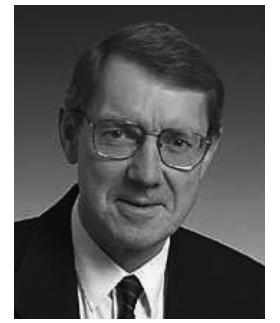

Palle Jeppesen (M'69) was born in 1941 in Vordingborg, Denmark. He received the M.Sc. degree in electrophysics, the Lic. techn. (Ph.D.) degree, and the Dr. techn. (D.Sc.) degree from the Technical University of Denmark (DTU), Lyngby, in 1967, 1970, and 1978 , respectively.

From 1968 to 1969, he was a Research Associate with Cornell University, Ithaca, NY, and, from 1969 to 1970, he was a Project Engineer with Cayuga Associates, Ithaca, NY; at both places, he did research in the field of GaAs Gunn effect microwave oscillators. From 1970 to 1998, he was an Assistant, Associate, Research, and Full Professor with the Department of Electromagnetic Systems (EMI), Technical University of Denmark, first in microwave electronics, and, since 1974, in optical communications. At EMI, he was Head of the Optogroup from 1974 to 1988 and Head of the Center for Broadband Telecommunications from 1988 to 1998 . From 1982 to 1984 , he also worked as a part-time Manager of R\&D at NKT Elektronik, now Draka Denmark Optical Cable, OFS Fitel Denmark, and Tellabs Denmark. Since 1998, he has been a Professor of optical communications with Research Center COM (now DTU Fotonik) where he is currently heading the Optical Transmission and Network Elements group and acting as coordinator of the five groups in the Communications Technology Research Cluster at DTU Fotonik. His current research interests are optical multi-level modulation formats and 160-640-Gbit/s optical communication. Currently, he is supervisor for six Ph.D. students.

Dr. Jeppesen was the recipient of the P.Gorm Petersens Memorial Stipend in 1974, the Esso Prize in 1978, the Villum Kann Rasmussen Prize in 1988 , and the Alexander Foss Gold Medal in 2005. He has been a member of numerous evaluation committees at the Technical University of Denmark and in other countries-in 2002 for Styrelsen för Strategisk Forskning, in 2003 and 2004 for Vetenskapsrådet and for Chalmers Tekniska Högskolan in Sweden, an in 2007 the peer-review panel for European Research Council Starting Grants and again for Styrelsen för Strategisk Forskning in Sweden. 